\title{
Caracterización socioeconómica de la producción cafetalera en la provincia de Rodríguez de Mendoza
} (Amazonas, Perú)

\section{Socioeconomic characterization of coffee production in the province of Rodríguez de Mendoza (Amazonas, Perú)}

\author{
Santos Triunfo Leiva-Espinoza ${ }^{1 *}$, Alcides Román Peña ${ }^{1}, K_{\text {Karol B. Rubio Rojas }}^{1}$
}

\section{RESUMEN}

El café es uno de los productos de exportación más importantes a escala mundial, ocupa el segundo lugar en comercialización detrás del petróleo. La presente investigación fue realizada en la provincia de Rodríguez de Mendoza en Amazonas, cuyo objetivo fue caracterizar las fincas cafetaleras instaladas en esta parte del país. La población con que se trabajó fue de 7061 productores y una muestra mínima de $n=104$ sobre la cual se aplicó una encuesta en la que se incluyeron preguntas relacionadas a aspectos socio económicos vinculados con la finca cafetalera. Los resultados mostraron que el $82,1 \%$ de productores de café tuvieron acceso a los servicios de salud, el $48,7 \%$ poseen agua entubada y tratada, así como el 70,1\% tuvo acceso a un desagüe articulado a la red pública. Se encontró también que al menos un $72,3 \%$ de los productores alcanzaron ingresos económicos por debajo del sueldo mínimo vital, sin embargo; se encontró un rendimiento de 16,03 qq/ha de café pergamino seco, que supera el promedio nacional; además se pudo observar que las fincas cafetaleras forman parte de un sistema diversificado y muy complejo, que involucra a los segmentos social, cultural, institucional y político.

Palabras clave: sistema de producción, finca cafetalera, Perú.

\begin{abstract}
Coffee is one of the most important export products on a world scale as it ranks second in marketing behind oil. The present investigation was carried out in the province of Rodríguez de Mendoza in Amazonas, whose objective was to characterize the coffee farms located in this part of the country. The population that worked was 7061 producers and a minimum sample of $\mathrm{n}=104$ on which a survey was applied which included questions related to socioeconomic aspects related to the coffee plantation. The results showed that $82.1 \%$ of coffee producers had access to health services, $48.7 \%$ had piped and treated water, and $70.1 \%$ had access to an articulated drain to the public network. It was also found that at least $72.3 \%$ of the producers achieved economic income below the minimum living wage, however; a yield of $16.03 \mathrm{qq} /$ ha of dry parchment coffee was found, which exceeds the national average; in addition it was observed that the coffee farms are part of a diversified and very complex system, involving the social, cultural, institutional and political segments.
\end{abstract}

Key words: production system, Characterization, Coffee, Peru. 


\section{INTRODUCCIÓN}

El sector cafetalero es el más importante de la agricultura peruana, y el café es considerado actualmente como el producto bandera de agro exportación (Alcántara et al., 2017), superando a Colombia y a Brasil (MINAGRI, 2017), aunque en el rubro cafés especiales esta ventaja es mínima. Ocupa alrededor de $400 \mathrm{mil}$ hectáreas de cultivo, que es el 8,6 \% de la superficie dedicada a la producción agrícola del país (Dilas y Cernaqué, 2017), se dice tambien que no solo es el principal producto de agro exportación sino incluye 120 mil hectáreas dedicadas a los cafés especiales que han conquistado los principales mercados de Europa y Norteamérica, representa el sustento de más de 230 mil familias y muchos más si incluimos a los integrantes de la cadena de producción. (MINAGRI, 2017). Tambien representa cerca de la mitad de las exportaciones agropecuarias y alrededor del 5\% del total de las exportaciones peruanas (Márquez et al., 2016), es generador de divisas y constituye fuente de ingresos para los productores (Vilca, 2014). La exportación de Café Grano Perú en el 2017 alcanza a Febrero los U\$ 58 millones a un precio promedio de U\$ 3,00 kilo donde USA sigue siendo el principal destino con U\$ 16 millones, le sigue Bélgica con U\$ 13 millones (AGRODATAPERU, 2017).

La importancia de la producción cafetalera, radica en el orden económico, social y ecológico (Palomino et al., 2014); convirtiéndose en una alternativa que puede mejorar la economía del productor campesino (Martínez et al., 2007)

El Perú actualmente no tiene una política económica que brinde, en su totalidad, ayuda a los productores y comercializadores del café orgánico peruano a comparación de Brasil y Colombia que han creado diversas políticas públicas las cuales apoyan a la mejora de la organización de los campesinos productores de café con el fin de desarrollar el concepto de marca colectiva y así mejorar la oferta exportable del producto. También se dice que la producción se realiza de manera artesanal (Solís, 2017).

Si bien es cierto la economía campesina depende en alto grado de los recursos, ciclos y fenómenos de la naturaleza, lo cual provoca que la producción se caracterice por la diversidad productiva, que es la única manera de amortiguar la impredecibilidad de buena parte de los fenómenos naturales y de evitar una dependencia excesiva de un mercado al que acuden en desventaja los campesinos (Cruz y Torres, 2016).

Los productores de la provincia la provincia de Rodríguez de Mendoza, región Amazonas tienen una gran diversidad de cultivos que contribuyen en el sustento familiar, pero el principal y generador de ingresos económicos, es el café. Si bien es cierto esta provincia tiene condiciones de clima y suelo adecuadas para este cultivo, esta región es el quinto departamento de mayor actividad cafetalera en Perú con más de 42744 hectáreas y representan el sustento de más de 26356 familias (MINAGRI, 2016).

Es necesario caracterizar sistemas socio productivos mediante diseños con enfoque agroecológico para incrementar la productividad del café y la estabilidad del agro ecosistema de la finca. Esto contribuiría a mejorar la calidad de vida de los agricultores que ahora se ve amenazada por la dependencia de unos pocos productos comercializables, con altos costos de producción y sujeto al vaivén de los precios en el mercado internacional. Un diseño agroecológico permitiría que las familias cafetaleras tengan mayor capacidad de adaptarse en un contexto de crisis socioeconómica del sector cafetero (Benítez et al,. 2015).

Resulta necesario conocer aspectos sociales relacionados a la producción cafetalera en Amazonas, considerando que el predominio del capital social en las tres regiones del país, indica que si se requiere orientar la política pública a la producción de café en especial orgánico en primer lugar se debería fortalecer la participación de los productores en organizaciones sociales de base (asociación, comité o cooperativa), además este tipo de organizaciones gozan de cierta ventaja comparativa en el ámbito de la producción orgánica (Mamani, 2015).

En este sentido, el presente trabajo se desarrolló con el objetivo de caracterizar los determinantes sociales y 
económicos de las fincas cafetaleras de la provincia de Rodríguez de Mendoza, Región Amazonas, el cual permitirá describir y analizar las principales variables sociales y económicas que caracterizan a dichas fincas.

\section{MATERIALES Y MÉTODOS}

La presente investigación se realizó en once distritos de la provincia Rodríguez de Mendoza ubicada a 1300 m.s.n.m con extensión territorial de 2359,39 km2 que representa el 20,35\% del área total del departamento de Amazonas (Figura 1). Está ubicada en la parte sur este de la región Amazonas a 60'3.98" y 6²3' 29.57" latitud sur y $77^{\circ} 7{ }^{\prime} 51.37^{\prime \prime}$ y $77^{\circ} 45^{\prime} 14.12^{\prime \prime}$ longitud oeste. La temperatura media anual es de $19^{\circ} \mathrm{C}$; presenta una precipitación de $1387 \mathrm{~mm} / \mathrm{año}$, su clima es por lo regular cálido húmedo, lo cual esta provincia de acuerdo a sus características geográficas representa un escenario apto para el desarrollo de la caficultura.

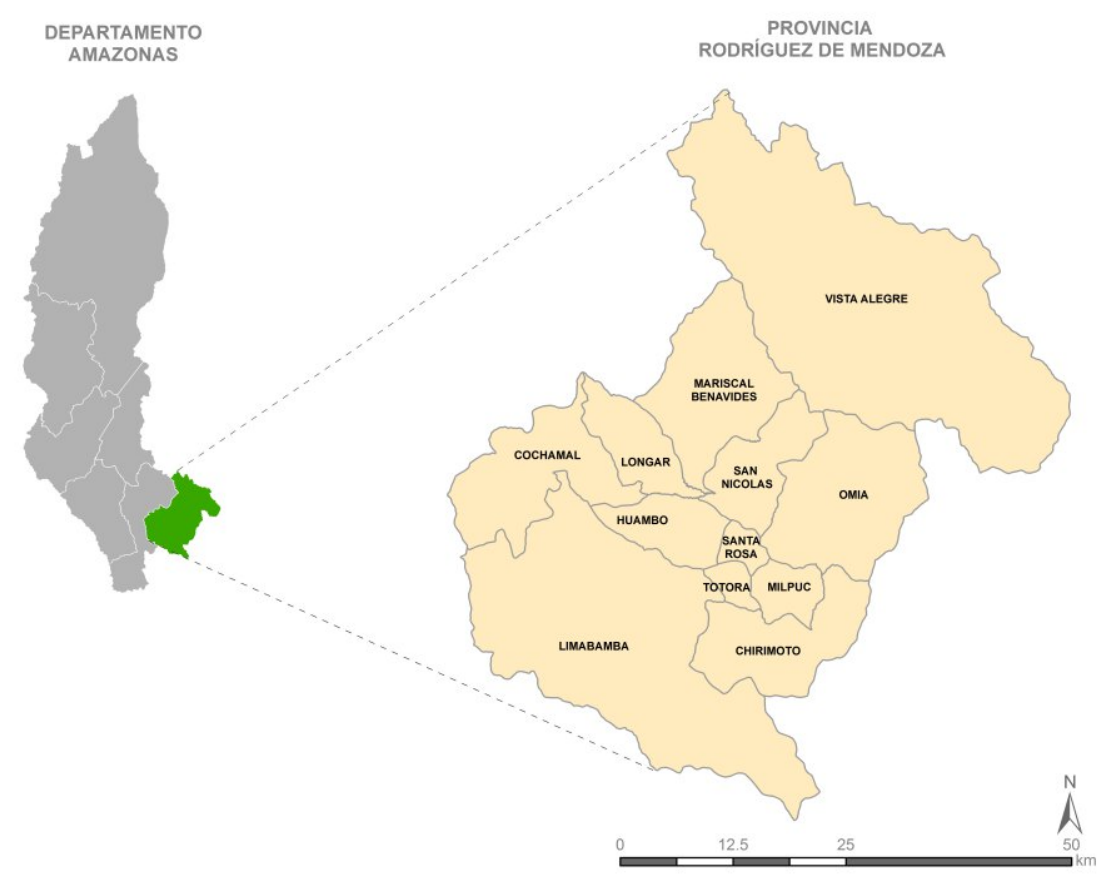

Figura 1. Ubicación geográfica del área de estudio.

Para la recopilación de información primaria se realizó elaboración, validación y la aplicación de encuestas, las mismas que constituyeron una herramienta de observación indirecta pues nos ha permitido captar los aspectos de la realidad a través de las palabras. Para su elaboración, se tomó como referencia la encuesta aplicada por Meneses (1999), así como también la información que se generó durante su investigación, esta herramienta se aplicó en el menor tiempo posible tomándose como tema central los aspectos a evaluarse de acuerdo a los objetivos del presente trabajo. Posterior a la elaboración de la encuesta, se procedió a la socialización de la misma; ésta se efectuó en dos oportunidades.
Las encuestas tuvieron una serie de preguntas que recopilaron información referente a: nivel de educación del productor, genero, edad del productor, relaciones internas del productor (pertenencia a alguna asociación, religión, etc.), la manera de aplicar la encuesta previamente ensayada y socializada fue mediante entrevistas individuales a un miembro de la unidad de producción que en la mayoría fue dirigida al jefe de la familia.

El tamaño de la muestra se calculó utilizando la fórmula de Schaeffer et al. (1987), estimándose un total de 104 encuestas dirigidas a productores cafetaleros jefes de familia. Se seleccionó una población objeto de la 
encuesta mediante un muestreo estratificado con afijación proporcional (todos los distritos estuvieron representados en la muestra

\section{RESULTADOS Y DISCUSIÓN}

Con respecto al género del responsable de la finca cafetalera, el 72,6\% fue del sexo masculino (Figura 2A). Por otro lado, el grupo etario más representativo (42,7\%) tuvo entre 41 y 60 años de edad, el menos representativo $(12,0 \%)$ lo constituyeron los productores jóvenes cuyas edades oscilaron entre los $18-25$
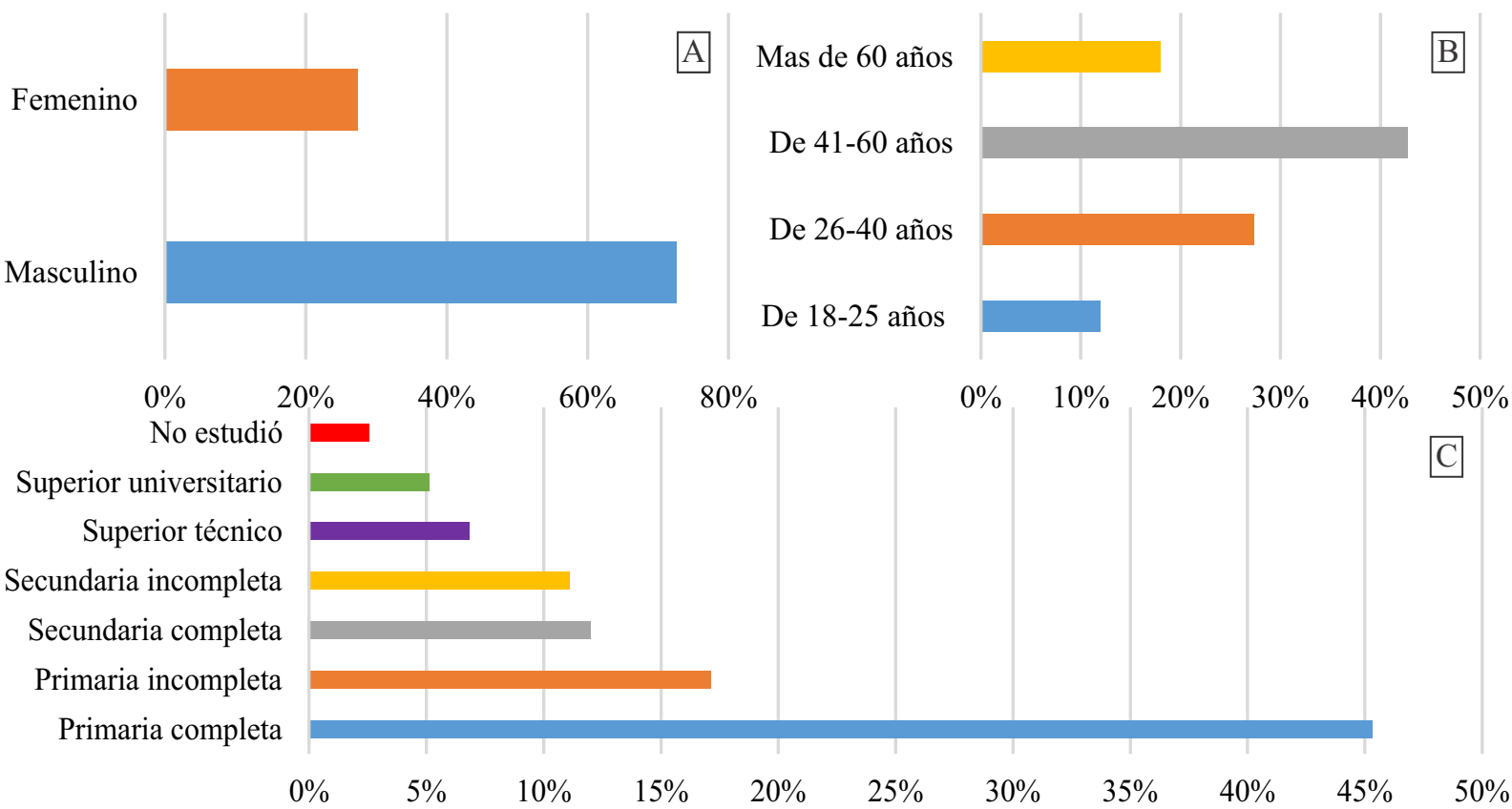

Figura 2. Características del productor encargado de la finca: sexo del productor encargado de la finca (B), edad del productor encargado de la finca (A) y grado de instrucción del encargado de la finca (C).

solamente el $9.2 \%$ corresponde al sector cafetalero.

Mientras que el mayor grupo de edad corresponde a adultos de entre 44 y 56 años, con un 35\%, seguido de 31 a 43 años, con 30.5\% (Junta Nacional del Café, 2016a). Por otro lado, el Instituto Nacional de Estadística e Informática INEI (2013) reporta que en el IV Censo Nacional Agropecuario CENAGRO 2012, en la región Amazonas el 81,1\% de productores agropecuarios corresponde al género masculino, lo cual guarda relación a lo encontrado en el presente trabajo; además, para la provincia de Rodríguez de Mendoza, el grupo etario más importante $(34,6 \%)$ lo componen los habitantes entre 25 y 39 años, aunque en su mayoría de los responsables de las fincas, se encuentran en un grupo de 40 a 59 años que representaría un 31,4\%; años de edad; un 27,4\% de productores responsables de las fincas tuvieron entre 26-40 años, completando el grupo con $17,9 \%$, los que tuvieron más de 60 años (Figura 2B). Además, la mayoría de entrevistados $(52,1 \%)$ refieren ser nativos del lugar, en tanto que el $47,9 \%$ fueron migrantes y provenientes de otras provincias y regiones del país. Los resultados se aproximan a lo encontrado en Cajamarca, Junín y Puno, en Perú, y en La Paz, Bolivia, aquí existió un bajo porcentaje de jóvenes, de entre 18 y 30 años de los cuales

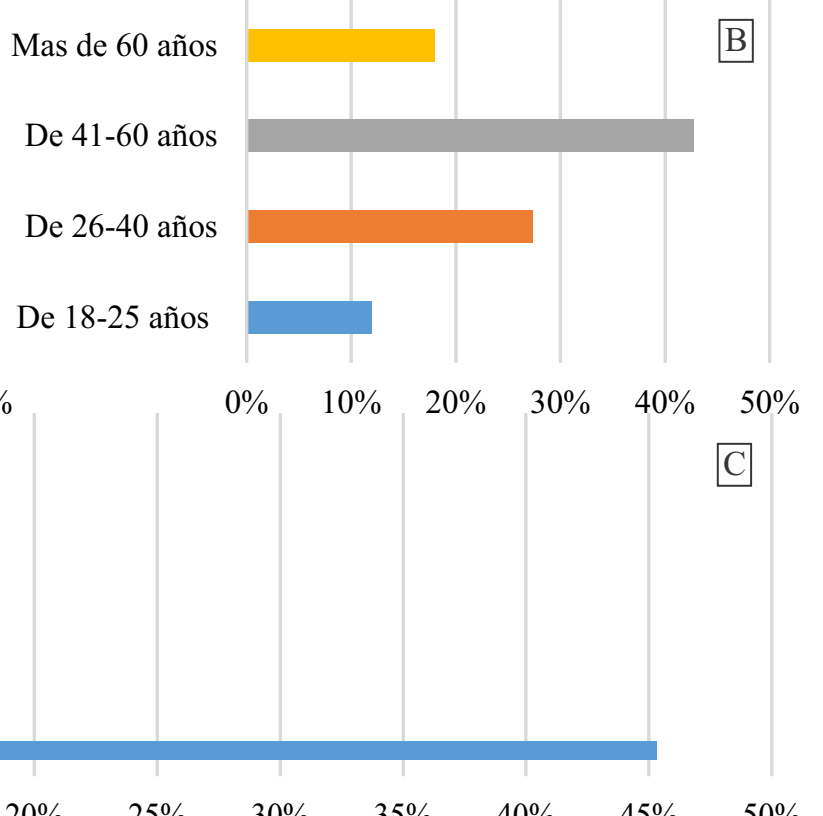

valor ligeramente inferior a lo encontrado en el presente trabajo.

Los productores cafetaleros de esta parte del país tienen un grado de instrucción, variable, la mayoría $(45,3 \%)$ solo cuenta con primaria completa, incluso existió un grupo considerable (17,1\%) que no terminó la primaria; similar es el caso de los productores con secundaria, donde se observa que solamente el $12 \%$ la concluyeron, mientras que un $11,1 \%$ inició sus estudios, pero no los concluyó. Por otro lado, y en referencia al nivel superior, se encontró que un 11,9\% cursaron estudios en institutos y la universidad, finalmente, un grupo minoritario de 3\% estudió el nivel inicial y solamente el 2.6\% no estudió (Figura $2 \mathrm{C}$ ). Al respecto, según el INEI (2013) informó que en la región Amazo- 
nas el 7,5\% de la población enmarcada entre los 15 años a más, es analfabeta, se muestra entonces que la tasa de analfabetismo en el ámbito de estudio es baja respecto a la tasa regional. Además, en el IV CENAGRO 2012 se reportó que el 7,9\% de productores agropecuarios no tienen ningún nivel educativo, mientras que $6,1 \%$ tienen educación superior no universitaria y universitaria. Estos valores guardan relación con lo encontrado en el presente estudio.

En referencia a los servicios básicos, si bien es cierto un $67,5 \%$ de productores tuvieron acceso a los servicios de salud a través del Seguro Integral de Salud del estado (SIS), además de que un porcentaje de 13,7\% de productores recibieron los servicios de salud del Seguro Social de Salud del Perú (EsSalud), todavía existe un notable $17,9 \%$ que no tiene acceso a este servicio; solamente un $0,9 \%$ de productores tiene acceso a servicios de salud privados (Figura 3A). Al respecto INEI (2015), reporta que para el 2015 un $71,8 \%$ de la población peruana contó con algún tipo de seguro donde el $40 \%$ fue atendido por el SIS y un 25,7 solo por EsSalud, además un 6,1\% contaba con otros seguros en la región; los resultados también guardan relación con lo reportado por la (Junta Nacional del Café, 2016b) en donde manifiestan que un 55,4\% de productores cafetaleros fueron atendidos en un centro de salud Público del país. Se visualiza un visible incremento del acceso a los servicios estatales de salud dentro de los dos últimos años, lo cual se debería a un notable impulso a las políticas de salud del gobierno peruano. (Wilson et al., 2009)

Por otro lado, el 94,9\% de productores cafetaleros tuvieron acceso al servicio de alumbrado eléctrico proveniente de una central hidroeléctrica, entre tanto un reducido $4,3 \%$ aún no cuenta con este servicio y además, existiría alrededor de $0,9 \%$ de productores que tienen acceso a energía eléctrica proveniente de otras fuentes como paneles solares y otras fuentes (Figura 3B). Los valores encontrados son similares a los reportados por el INEI (2015) donde se menciona que para el primer semestre del 2015 el 94,2\% de hogares contó con alumbrado eléctrico por red pública.

En cuanto a los servicios de agua y desagüe, si bien es cierto, un 70,1\% de los productores cuenta con desagüe articulado a la red pública, un grupo de 29,9\% aún no lo tiene, aunque de este grupo, el 11,1\% cuenta con letrina, el $10,3 \%$ con pozo séptico y un $0,9 \%$ utiliza otro tipo de desagüe, finalmente el $7,7 \%$ de productores no contó con ningún tipo de desagüe (Figura 3C). En cuanto al servicio de agua, el 48,7\% de entrevista-

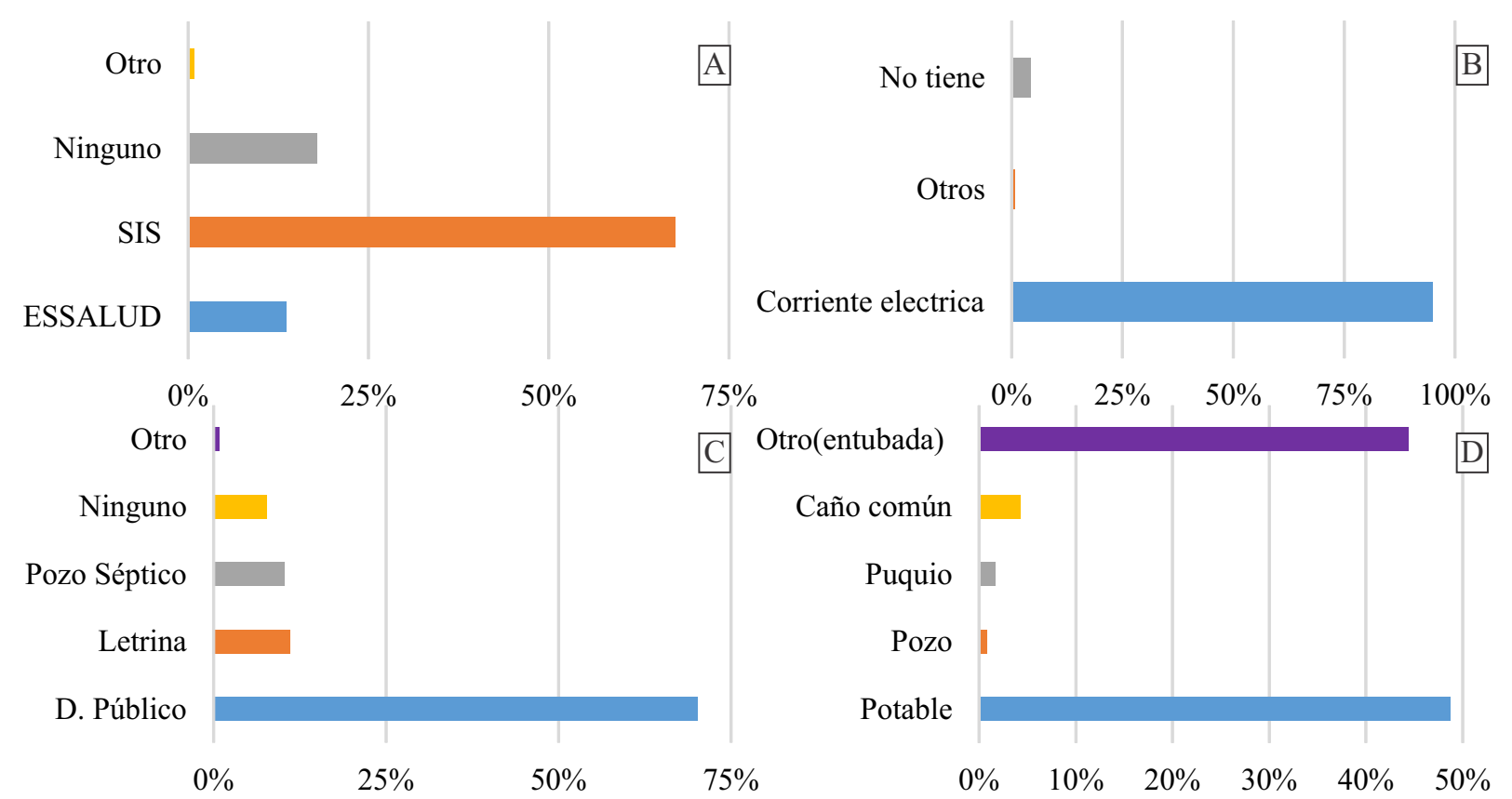

Figura 3. Servicios básicos con los que cuenta la finca y el productor: Salud (A), Luz(B), Desagüe (C) y Agua (D) 
dos refirieron haberlo recibido entubada y tratada, el $44,4 \%$ lo recibió entubada y sin tratamiento alguno, mientras que el 4,3\% usaron un grifo en común y finalmente el 2,6\% de productores toma agua de un puquio o pozo (Figura 3D). Estos resultados indican que existe un notable incremento de este servicio desde el reporte efectuado por el INEI (2015) en donde se menciona que el $85.8 \%$ de los hogares del país se abastecen de agua proveniente de una red pública; por otro lado y específicamente en Amazonas el porcentaje fue menor (79,1\%); Lo encontrado guarda relación con lo reportado por el INDES-CES (2016), quienes refieren que durante los últimos cinco años, en la provincia de Rodríguez de Mendoza, ha existido diferentes proyectos de saneamiento rural que han contribuido a mejorar los servicios de agua, desagüe y energía eléctrica; $\sin$ embargo estos aún podrían ser escasos para un grupo representativo de esta población.

Para comunicarse, el 99,1\% de productores utilizó teléfono celular personal o la de un familiar, mientras que el 0,9\% restante utilizó teléfono fijo; la población de esta parte del país, utiliza variadas formas de comunicación e información, así tenemos que todos usan más de un medio, por ejemplo, el 56,1\% utiliza Tv + radio, un segundo grupo de $32,3 \%$ sólo utiliza TV y finalmente un tercer $(11,6 \%)$ grupo utilizó solamente radio. Al respecto el INEI (2015) reporta que el 87,2\% de hogares del país cuenta con al menos un miembro que hace uso de teléfono celular, así mismo, en la localidad de Jipijapa en Manabí- Ecuador se reporta una realidad similar pues los cafetaleros de esta localidad usan medios de comunicación y en si mayoría (47\%) usa la TV + folletos + radio, un segundo grupo (25\%) usa $\mathrm{TV}+$ folletos + radio + celular, un tercer grupo $(20 \%)$ usa TV + folletos + radio + periódico + celular. (Santiestevan et al., 2014)

El 79,9\% de fincas de la provincia estarían interconectadas hacia carreteras afirmadas que en épocas secas estarían en buen estado, aunque el 17,5\% de parcelas no tendrían un acceso normal en épocas lluviosas, situación que dificultaría la comunicación terrestre hacia los centros de acopio de los productos. Por otro lado, se encontró que la mayoría de productores $(55,5 \%)$ vive en la finca sin embargo; un elevado $45,5 \%$ no lo hace y tiene que transportar sus cosechas hacia su lugar de residencia; en este sentido se encontró que el $85,9 \%$ de productores traslada sus productos desde la chacra hasta su domicilio en lomo de bestia, de este grupo, en su mayoría (46,5\%) demora menos de una hora, el 29,7\% demoraría hasta dos horas, un $13,5 \%$ tarda de dos a tres horas y un 10,3\% tardaría más de tres horas para transportar sus productos de la finca hacia su domicilio. Situaciones similares se reportaron en otros países como México (Estado de Chiapas) en donde el 49,4\% de unidades productivas de la región estaban conectadas por carreteras de tierra que, a diferencia de las épocas secas, en las de abundante lluvia se tornan inaccesibles (Medina et al., 2014).

En referencia a las actividades económicas de los productores cafetaleros, un grupo mayoritario de 93,2\% de los entrevistados mencionaron tener como actividad económica principal a la agricultura, mientras que un 5,1\% combina a la agricultura y la ganadería como actividades principales, y solamente el 1,7\% se dedican a otras actividades. Según el INEI (2013), en la región Amazonas, el 67.7\% de productores se dedican a la agricultura, ganadería y pesca, lo encontrado en el presente trabajo confirma que la agricultura es el principal sustento económico de las familias asentadas en esta parte del país. Por otro lado, quienes se dedican a la práctica de la actividad principal son los jefes de familia (43,6\%), los jefes de familia y sus esposas $(41,9 \%)$ así como la esposa por si sola y los hijos en un $7,7 \%$ y $6 \%$, respectivamente, finalmente se encontró que en un $0,9 \%$ de los casos son otras personas, ajenas al núcleo familiar, las que se dedican a esta actividad. Al respecto; se muestra a la mujer como un componente activo dentro del hogar, pues además tuvieron una participación de un $86.9 \%$ en trabajos vinculados a la producción de café, estos y otros trabajos adicionales a las labores de ama de casa dan fe de la importancia del género femenino en el sistema productivo del café; estos resultados concuerdan con la 
Junta Nacional del Café (2015), cuando menciona que más del 30\% de unidades familiares están siendo conducidas por las mujeres.

Respecto a los ingresos económicos de los productores cafetaleros de Rodríguez de Mendoza, un grupo mayoritario de $72,3 \%$ refiere que tuvo un ingreso mensual entre US\$100,00 a 250,00 dólares, el segundo grupo (12,5\%) tuvo un ingreso mensual entre los US\$251,00 y US\$ 300,00. por otro lado, un 9.3\% tuvo ingresos entre US\$ 301,0 a US\$ 400,00 dólares, también un $5,9 \%$ de productores obtuvieron ingresos mensuales entre US\$ 401- US\$ 500, 00 finalmente, un grupo conformado por un 6,5\% alcanzó ingresos mensuales mayores a US\$. 500,00 pero menor a US\$. 1200,00. En este sentido, aun cuando el café sería una importante herramienta para dejar la pobreza, tener acceso a los servicios básicos y a una buena educación (Junta Nacional del Café, 2016c); los resultados no son tan halagadores y muestran que al menos un $72,3 \%$ de los productores tendrían ingresos económicos por debajo del sueldo mínimo vital que en el Perú es de $\mathrm{S} / .850 .00$ soles mensuales, este grupo mayoritario formaría parte de un sistema económico de subsistencia o de autoconsumo, considerando que el 93,7\% de entrevistados refirieron producir y consumir cultivos de pan llevar que producen en su finca familiar. Además, en su mayoría de encuestados (69,6\%) manifestaron que no es posible ahorrar con fines previsionales, manifestando además que no cuentan con ningún tipo de jubilación $(88,7 \%)$ por ello deberán trabajar hasta que sus fuerzas les permita. Lo encontrado tiene alguna semejanza con los resultados encontrados por Santistevan et al., (2014), quien encontró que en Jipijapa (Ecuador), el nivel de ingresos del productor cafetalero es bajo, determinando que los ingresos económicos de al menos el $44 \%$ de productores no alcanza siquiera una remuneración básica unificada; se señala además que al igual que en otros países, el ingreso por el café es estacional (se percibe ingresos solamente en época de cosecha), lo que resulta insuficiente para el sustento de la familia; aunque los cafetaleros, además de este producto también se dedican a otros tipos de cultivos como plátanos, yuca, frutales y crianza de animales menores, que venden para obtener ingresos económicos adicionales a los obtenidos por la venta del café; aunque se evidencia una alta tendencia al autoconsumo de los cultivos anuales que producen los productores cafetaleros, por ejemplo en el Cusco los cultivos permanentes se destinan mayormente para la venta ( $15 \%$ al autoconsumo); los cultivos anuales en cambio, se consumen más en la propia zona ( $80 \%$ de autoconsumo); la misma tendencia tienen las crianzas (75\% destinados al autoconsumo)(Merma y Julca , 2012). En este sentido, Al productor le interesa asociar o instalar varios cultivos en su finca, de acuerdo a una estrategia de diversificación para reducir impactos que podría hacer peligrar el ingreso económico de sus familias (Merma y Julca, 2012). La producción de alimentos a pequeña escala puede ser una alternativa para una adecuada alimentación en el hogar además de aumentar los ingresos por la vía de la comercialización y el autoabastecimiento (Álvarez et al., 2007).

La productividad del café en el Perú es baja, entre otros factores por falta de asistencia técnica y capacitación (Junta Nacional del Café, 2016d); el presente estudio así lo confirma, aun cuando se encontró que el 59,0\% y $56,4 \%$ de productores accedieron a al menos a un servicio de capacitación y asistencia técnica, respectivamente; las entidades que prestaron mayor servicio de extensión fueron las privadas y las propias organizaciones con $83,8 \%$ y $87,5 \%$ en capacitaciones y asistencia técnica respectivamente, el Servicio Nacional de Sanidad Agraria (SENASA) capacitó al 22,1\% de productores y a un $14,1 \%$ prestó servicio de asistencia técnica, el Gobierno regional aportó con un reducido $5,9 \%$ y $6.3 \%$ para capacitación y asistencia técnica, respectivamente; la Universidad Nacional Toribio Rodríguez de Mendoza de Amazonas (UNTRM) aportó también con 5,9\% de capacitados, al igual que los Organismos No gubernamentales (ONG); finalmente completan el grupo, las Municipalidades, con un reducido $2,9 \%$ y $1,6 \%$ para capacitación y asistencia técnica, respectivamente. Al respecto, el INEI (2013) menciona que las asociaciones, 
comités y cooperativas de productores constituyen las instituciones que son reconocidas como las que mayormente ofrecen servicios de capacitación, asistencia técnica y asesoría empresarial a los productores agropecuarios.

Con respecto a la pertenencia a organizaciones, se encontró que un elevado $42,7 \%$ de productores no se encuentra asociados a algún gremio cafetalero; el $14,5 \%$ se articula a la empresa privada, el 12,0\% están articulados a la Cooperativa de Productores Agropecuarios Rodríguez de Mendoza (COOPARM), mientras que un 30,8\% se encuentra vinculado a otros gremios cafetaleros. Los resultados son similares a los reportados por la Junta Nacional del Café (2015), quienes manifiestan que el café sirve de sustento para 223 mil familias a nivel nacional, pero menos de la mitad está organizada en asociaciones o cooperativas de producción; es así que la cooperativa cafetalera COOPARM viene mostrando un rol protagónico en esta provincia y ya desde años anteriores se reconoce su importancia dentro del sector cafetalero de la región y el país, (Dirección Regional Agraria Amazonas, 2011)

\section{CONCLUSIONES}

De los resultados obtenidos en la presente investigación se diferencian las características y los problemas que viene atravesando el sector cafetalero de la provincia de Rodríguez de Mendoza en la región Amazonas; en este sentido se concluye que las fincas son diversificadas, aunque la producción de café es la actividad principal y representa el principal ingreso económico de los productores asentados en esta parte del país. Se logró diferenciar que a la fecha se ha producido un visible incremento en la mejora de los servicios básicos como agua, desagüe, electricidad y medios de comunicación, sin embargo; éstos aún serían escasos para un importante sector de la población.

Por otro lado, además se contempla que el sistema productivo del café se asemeja a la realidad productiva nacional y de Latinoamérica; con visibles falencias en los niveles de producción y comercialización de café; aunque con ligeros indicios de haberse iniciado en un proceso de transición o migración hacia un eslabón tecnológico mayor. El sector cafetalero requiere una atención especial que involucre un cambio estructural dentro de las políticas públicas nacionales, requiriéndose mayor compromiso y participación en el sector cafetalero y que contribuya así a prestar mayor atención a los pequeños agricultores quienes están en condiciones de mayor desventaja para mejorar su capacidad productiva y de organización.

\section{REFERENCIAS BIBLIOGRÁFICAS}

Agrodataperu. 2017. Café Perú Exportación 2017 febrero. Lima (Perú). Recuperado de: https://www. agrodataperu.com.

Alcántara, C., B. Efadias, G. E. Gallardo Camarena de Huisa y N. S. Morales Galiano de Mestanza. 2017. Planeamiento estratégico del sector cafetalero peruano. Tesis de Maestría. Pontifica Universidad Católica del Perú. Lima (Perú).

Álvarez, M. C., M. López, L. P. Cortés y J. E. Torres. 2007. "Caracterización socioecómica y seguridad alimentaria de los hogares productores de alimentos para el autoconsumo, AntioquiaColombia". Agroalimentaria 25: 109-122.

Benítez E., J.L. Jaramillo, S. Escobedo y S. Mota. 2015. "Caracterización de la producción y del comercio de café en el municipio de Cuetzalan, Puebla”. Agricultura, Sociedad y Desarrollo 12: 181-219.

Cruz, S. y G. Torres. 2016. “Caracterización del agroecosistema cafetalero en la Sierra Sur de Oaxaca”. En Química, Biología y Agronomía. Handbook T-I. Rérez, F., E. Figueroa, L. Godínez, J. Quiroz y R. García (eds.). Texcoco (México): ECORFAN.

Dilas, J. y O. Cernaqué. 2017. El sector cafetalero peruano: Un enfoque a la CTI para su competitividad. Huancayo (Perú): Universidad Continental.

DRAA (Dirección Regional Agraria). 2011. Plan 
estratégico regional agrario de la región Amazonas 2011-2021. Chachapoyas (Perú): DRAA. Recuperado de: http://www.region amazonas.gob.pe.

INEI (Instituto Nacional de Estadística e Informática). 2013. IV Censo Nacional Agropecuario: Amazonas perfil agropecuario. Lima (Perú): Punto y Grafía.

INEI (Instituto Nacional de Estadística e Informática). 2015. Perú: Sintesis estadística 2015. Lima ( Perú ): INEI. Recuperado de: https://www.inei.gob.pe/.

Junta Nacional del Café. 2015. "Promueven acceso de cafetaleros a sistemas de protección social". El cafetalero 13:26-27.

Junta Nacional del Café. 2016a. “Campaña cafetalera se inició con mejor cosecha, pero bajos precio". El cafetalero 14: 10-12.

Junta Nacional del café. 2016b. "Expo Café Perú, alianza de productores, industria y Estado, para la competitividad". El cafetalero 14: 4-9.

Junta Nacional del Café. 2016c. "Quintal de datos: Cifras sociales preocupantes". El Cafetalero $14: 6-8$.

Junta Nacional del Café. 2016d. "Una alianza por la calidad en la caficultura peruana". El cafetalero $14: 20-25$

Mamani, J.W.T. 2015. “Caracterización socioeconómica y ambiental de la producción de café orgánico en el Perú”. Revista de Investigaciones Altoandinas 17:23-32.

Martínez M.Á., V. Evangelista, F. Basurto, M. Mendoza y A. Cruz-Rivas. 2007. "Flora útil de los cafetales en la Sierra Norte de Puebla, México". Revista mexicana de biodiversidad 78: 15 40.

Márquez, F, A. Julca, M. Cantom, H. Soplín, S. Vargas y P. Huerta. 2016. "Sustentabilidad Ambiental En Fincas Cafetaleras Después De Un Proceso De Certificación Orgánica En La Convención (Cusco, Perú)". Ecología Aplicada 15: $125-$
Meneses, M. 1999. Enfoque metodológico mixto aplicado al diseño de encuestas para el estudio de la participación política. Monterrey (Mexico): Tecnológico de Monterrey.

Merma, I. y A. Julca. 2012. "Caracterización y evaluación de la sustentabilidad de fincas en Alto Urubamba, Cusco, Perú”. Ecología aplicada 11:1-11.

MINAGRI (Ministerio de Agricultura y Riego). 2016. AMAZONAS: Cosechando desarrollo en la tierra de los Chachapoyas: resultados 20112016. Chachapoyas (Perú): MINAGRI.

MINAGRI (Ministerio de Agricultura y Riego). 2017. Este año superaremos los US\$800 millones en exportaciones de café. Lima (Perú): MINAGRI.

Palomino, C., C. López, R. Espejo, R. Mansilla y J. Quispe. 2014. "Evaluación de la diversidad genética del café (Coffea Arabica L.) En Villa Rica(Perú)". Ecología aplicada 13: 129-234.

Schaeffer, R. L., W. Mendehall y L. Ott. 1987. Elementos de muestreo. México D.F. (México): Grupo Editorial Iberoamérica.

Solís, L. S. 2017. “Análisis comparativo de las políticas públicas para promocionar internacionalmente el café en los países sudamericanos". San Martín Emprendedor 4: 34-43.

Vilca, S. R. O. Evaluación de la influencia de parámetros de fermentación en la calidad sensorial del café (Coffea arabica L.) del valle Inambari-Sandia. Tesis de Grado. Universidad Nacional del Altiplano. Puno(Perú).

Wilson L., A. Velásquez y C. Ponce. 2009. “La ley marco de aseguramiento universal en salud en el Perú: Análisis de beneficios y sistematización del proceso desde su concepción hasta su promulgación”. Revista Peruana de Medicina Experimentaly Salud Publica 26:207-217. 